\title{
The optical depth including Lorentz invariance violation energy threshold shifts
}

\author{
Humberto Martínez-Huerta*a $\dagger$ \\ E-mail: humbertomheifsc.usp.br
}

\section{Rodrigo G. Lang a}

E-mail: rodrigo.lang@usp.br

\section{Vitor de Souza ${ }^{\text {a }}$}

E-mail: vitoreifsc.usp.br

${ }^{a}$ Instituto de Física de São Carlos, Universidade de São Paulo,
Av. Trabalhador São-carlense 400, São Carlos, Brasil.

\begin{abstract}
Lorentz invariance violation (LIV) introduced as a generic modification to particle dispersion relations can change the photon energy threshold of pair-production, which modifies the expected gamma-ray flux from astrophysical sources. In this work, we review this phenomenon and explore its consequences through the derived effects in the optical depth. Then, by looking for subluminal LIV signatures in TeV gamma-ray spectra, we present stringent limits to the LIV energy scale at leading order $n=1$ and 2. And finally, we present the predicted flux of GZK-photons including LIV, in the astrophysical scenario which best describes UHECR data.
\end{abstract}

International Conference on Black Holes as Cosmic Batteries: UHECRs and Multimessenger Astronomy BHCB2018

12-15 September, 2018

Foz do Iguaçu, Brasil

\footnotetext{
${ }^{*}$ Speaker.

${ }^{\dagger}$ The authors acknowledge FAPESP support numbers 2015/15897-1, 2016/24943-0 and 2017/03680-3. The authors also acknowledge the National Laboratory for Scientific Computing (LNCC/MCTI, Brazil) for providing HPC resources of the SDumont supercomputer, which have contributed to the research results reported within this paper (http://sdumont.lncc.br).
} 


\section{Introduction}

The precise measurements of cosmic messengers from very energetic phenomena in the Universe, have set an unprecedented opportunity to test fundamental physics. Among the possibilities, to test and explore the limits of validity of the Lorentz symmetry has been an important motivation for theoretical and experimental research. Moreover, some Lorentz invariance violation (LIV) has been proposed by quantum gravity and effective field theories [1-12]. Therefore, astroparticle signatures of LIV derived phenomena in the photon sector have been searched through the arrival energy time delays, photon splitting, spontaneous photon emission, shifts in the pair production energy threshold and many others effects [13-41]. Following this line of thought, in the present proceeding we review and show some recent results of our LIV signatures searches in cosmic and gamma rays. In Section 2, we present the phenomenological generalization of the LIV-induced modifications to the particle dispersion relation. Then, we review and explore the scenarios when LIV is considered in the electron-positron production by a high energy photon that interacts with some low energy background light and show that the LIV effect is a shift to the minimum energy that the pair-production process requires, we present this in Section 2.1. The consequence of such effects, in the so called subluminal LIV-scenarios, is the increase of the optical depth at given energy regions, which, as we illustrate in Section 3, are sensitive to the amount of LIV. The latter predicts that more photon events can be expected in different energy windows than in a Lorentz invariant (LI) scenario. Thus, in Section 3.1, we present results of a LIV signature search in $\mathrm{TeV}$ gamma-rays. Then, in Sec. 3.2, we show the expected, LI and LIV, EeV photon flux produced by the decay of the secondary cosmic ray flux of neutral pions, the usually named GZK-photons. Finally, in Section 4, some conclusions and remarks are presented.

\section{Lorentz invariance violation}

A phenomenological generalization of the LIV-induced modifications to the dispersion relation formalism converges to the introduction of an extra term in the $a$-specie particle dispersion relation, which, among other possibilities, can be motivated by the introduction of a not explicitly Lorentz invariant term in the free particle Lagrangian $[14,16]$ or by some spontaneous Lorentz symmetry breaking [2]. So that, the corrected dispersion relation becomes

$$
E_{a}^{2}-p_{a}^{2}=m_{a}^{2} \pm\left|\delta_{n, a}\right| E_{a}^{(n+2)}
$$

where $\left(E_{a}, p_{a}\right)$ stands for the four-momenta of the $a$-particle type with mass, $m_{a}$. For simplicity, natural units are used in this work, unless a different one is explicitly given. The $( \pm)$ sign, characterize the so called superluminal (+) and subluminal (-) dominant phenomena due to $\delta_{n, a}$, which is the Lorentz invariance violation parameter, where $n$ express the leading order of the correction. $\delta_{n, a}$ is frequently considered to be inversely proportional to some LIV energy scale M, to suppress higher-dimension operators with some coefficients given by the underlying theory, and it is also common to associate $\mathrm{M}$ with the Plank energy scale $\left(\sim 10^{19} \mathrm{GeV}\right)$. However, without loss of generality, the LIV term can be named $E_{\mathrm{LIV}}^{(n)}=1 /\left|\delta_{n}\right|^{1 / n}$ for $n>0$, as we do in Section 3.1.

It has been shown in previous works that processes of photo production can lead to new physics when LIV is considered through Eq. 2.1, and there is some shift of the minimum energy that these 
processes need to be kinematically allowed [17, 18, 22, 23, 32], which it is discussed in the next Section.

\subsection{Pair production energy threshold shifts}

In the particular scenario where the interaction of very energetic particles, such as cosmic and gamma rays, propagating through the background light of the Universe, LIV can lead to measurable signatures in the observed cosmic particle spectra, due to changes in the expected effects of relevant processes of photon-particle production, such as photo pion-production and pair-production processes. Hereafter, we focus in the latter due to its strong impact as an energy lose mechanism in the propagation of very high energy photons. Although, other processes such as the Compton scattering can also lead to LIV signatures that modify the observed photon energy spectrum, it is noted in Ref. [40], that its effects are small in comparison with those expected by photo pair production.

The derived physics from Eq. 2.1 leads to shifts at the minimum energy that these processes needs to be kinematically allowed, which can change the expected photon flux from distant sources, as we explore in the next Sections. In the case of the pair-production process ${ }^{1}, \gamma \gamma_{b} \longrightarrow e^{+} e^{-}$, if LIV effects are considered for photons and leptons, Eq. 2.1 takes the form

$$
E_{\gamma}^{2}-p_{\gamma}^{2}=\delta_{n} E_{\gamma}^{n+2}, \quad \text { and } \quad E_{ \pm}^{2}-p_{ \pm}^{2}=m_{e}^{2}+\delta_{ \pm, n} E_{ \pm}^{n+2}
$$

where sub-indices $\gamma,+$ and - , denote photons, positron and electron species respectively. Due to the very high energy of the gamma-rays that we are considering, hereafter, we take $E_{\gamma} \gg m_{e}, \varepsilon$, thus, the kinematics of the process for a head-on collision, with inelasticity $K,\left(E_{+}=K\left(E_{\gamma}+\varepsilon\right)\right)$, satisfies the following expression [32],

$$
\varepsilon=\frac{m_{e}^{2}}{4 E_{\gamma} K(1-K)}-\frac{1}{4} \delta_{n}^{t o t} E_{\gamma}^{n+1} ; \quad \delta_{n}^{\text {tot }}=\delta_{\gamma, n}-\delta_{+, n} K^{n+1}-\delta_{-, n}(1-K)^{n+1} .
$$

Notice that $\delta_{n}^{\text {tot }}$ is a linear combination of the LIV contributions from the different particle species. Also note that, if LIV is such that, $\delta_{n}=\delta_{\gamma, n}=\delta_{ \pm, n}$, for the scenario with $K=1 / 2$,

$$
\delta_{n}^{\mathrm{tot}}=\left(1-\frac{1}{2^{n}}\right) \delta_{n}
$$

On the other hand, when LIV is considered only (or dominated by) the photon sector, i.e. $\delta_{ \pm}=0$ (or $\delta_{\gamma} \gg \delta_{ \pm}$), then, $\delta_{n}^{\text {tot }}=\delta_{\gamma, n}$ (or $\approx \delta_{\gamma, n}$ ). Therefore, there is only a factor of $\left(1-\frac{1}{2^{n}}\right)$ between this two set of scenarios. So that, for simplicity and as in previous works, these second scenarios are considered in Sections 3.1 and 3.2, which also allows the particular case of LIV at $n=0$.

Solutions for one of the photon energies in Eq. 2.3 are the photon energy thresholds, $E_{\gamma, t h}$ or $\varepsilon_{t h}$, that turns on the process. Solving for $E_{\gamma, t h}$, Eq. 2.3 is a polynomial equation of order $n$ that has three main scenarios depending on $\delta_{n}^{\text {tot }}$. a) If $\delta_{n}^{\text {tot }}=0$, the standard LI-threshold is recovered, which correspond to the central panel in Fig.1, where pair-production is allowed for any $E_{\gamma}$ once the gamma-ray energy reaches the grey regions. As an example, in the Figure 1 there are different areas marked for different background temperatures, $0.5,1,1.5,2$ and $2.7 \mathrm{~K}$, from lighter to darker grey, where each shaded area includes the previous ones. However, as pointed in Ref. [32], if the

\footnotetext{
${ }^{1}$ Sub-index $b$ indicates the low energy photons from the background light with energy $\varepsilon$.
} 

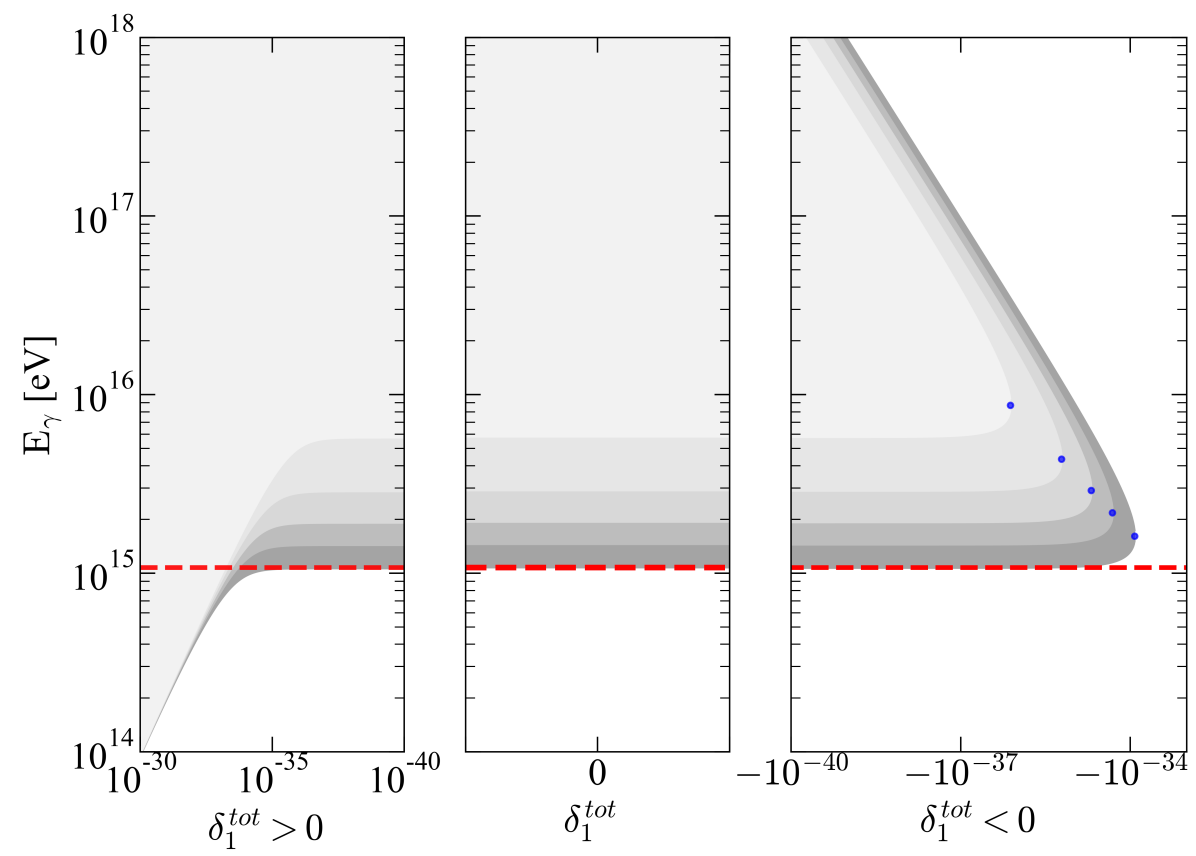

Figure 1: The three different scenarios when LIV effects are considered in the particular case of, $\gamma \gamma_{b} \rightarrow e^{+} e^{-}$and LIV leading order $n=1$. Grey regions show the pair-production allowed configuration at background temperatures of $0.5,1,1.5,2$ and $2.7 \mathrm{~K}$, from lighter to darker greys, where each shaded area includes the previous ones. Similar behaviour can be expected for different temperatures and leading orders $n$, but different energies. At the central panel, the LI scenario, $\delta_{n}^{\text {tot }}=0$, thus, $E_{\gamma, t h}^{\mathrm{LIV}}=E_{\gamma, t h}^{\mathrm{LI}}$. In the left panel, there are the superluminal scenarios, $\delta_{n}^{\text {tot }}>0$, where the LIV energy threshold is $E_{\gamma, t h}^{\mathrm{LIV}} \leq E_{\gamma, t h}^{\mathrm{LI}}$. At the right panel, there are the subluminal scenarios, $\delta_{n}^{\text {tot }}<0$, where energy threshold shift is such that $E_{\gamma, 1, t h 1}^{\mathrm{LIV}} \geq E_{\gamma, t h}^{\mathrm{LI}}$ and the pair production is constrained to a region $E_{\gamma, 1, t h 1}^{\mathrm{LIV}} \geq E_{\gamma} \leq E_{\gamma, 1, t h 2}^{\mathrm{LIV}}$, which gets shorter as the LIV effect increases, until it reaches a critical point (in blue), where no process is allowed at all. Contrary to the previous scenario, more gamma-rays can be expected. The red dotted line is the $E_{\gamma, t h}^{\mathrm{LI}}$ at $2.7 \mathrm{~K}$ for comparison.

LIV parameter is not zero, there are two more scenarios depending on the sign of $\delta_{n}^{\text {tot }}$. b) If $\delta_{n}^{\text {tot }}>0$, then $E_{\gamma, t h}^{\mathrm{LIV}} \leq E_{\gamma, t h}^{\mathrm{LI}}$, that is, there is a threshold shift to lower energies as displayed in the left panel of Fig. 1. For comparison, the red dotted line show the $E_{\gamma, t h}^{\mathrm{LI}}$, at $2.7 \mathrm{~K}$. c) If $\delta_{n}^{\text {tot }}<0$, the so called "recovery scenarios", there is a threshold shift to higher energies and the process is allowed only in the region $E_{\gamma, t h_{1}}^{\mathrm{LIV}}<E_{\gamma}<E_{\gamma, t h_{2}}^{\mathrm{LIV}}$, as shown in the right panel of Fig. 1. Moreover, if $\left|\delta_{n}^{\text {tot }}\right|>\left|\delta_{n}^{c r}\right|$, the LIV effect is so strong that no pair-production is allowed at all. These critical points are indicated with the blue points in Fig. 1 and given by

$$
E_{\gamma}^{c r}(\varepsilon ; n)=\frac{n+2}{n+1} \frac{m_{e}^{2}}{4 K(1-K)} \frac{1}{\varepsilon}, \quad \delta_{n}^{c r}(\varepsilon ; n)=\frac{(n+1)^{n+1}}{(n+2)^{n+2}}\left(\frac{4 K(1-K)}{m_{e}^{2}}\right)^{n+1} 4 \varepsilon^{n+2} .
$$

In the next Section, we explore the implications of gamma-rays interacting with the different dominant background lights in different energy regions of interest. 


\section{The Optical Depth including LIV}

As pointed out, very high gamma-rays that propagate from distant sources suffer significant attenuation due to pair-production, which constrains how far in the universe we, on Earth, can expect photons without being absorbed, which is the optical depth [42]. Therefore, the effect of shifting the energy threshold of pair-production leads to a change in the LI-expected gamma-ray flux.

It has been shown in previous works (see for instance Refs. $[31,35-37,41]$ ), that the optical depth including LIV effects can be obtained by

$$
\tau_{\gamma}\left(z, \theta, \eta_{b}, E_{\gamma} ; n, \delta_{n}^{\mathrm{tot}}\right)=\int_{0}^{z} \mathrm{~d} z \frac{c}{H_{0}(1+z) h(z)} \int_{-1}^{1} \mathrm{~d}(\cos \theta) \frac{1-\cos \theta}{2} \int_{\mathcal{\varepsilon}_{t h}}^{\infty} \mathrm{d} \varepsilon \eta_{b}(\varepsilon, z) \sigma\left(E_{\gamma}, \varepsilon, z\right),
$$

where $H_{0}=70 \mathrm{~km} \mathrm{~s}^{-1} \mathrm{Mpc}^{-1}$ is the Hubble constant, $c$ is the speed of light in vacuum, $h(z)=$ $\sqrt{\Omega_{\Lambda}+\Omega_{M}(1+z)^{3}}$ is the distance element in a expanding universe with, $\Omega_{\Lambda}=0.7$ and $\Omega_{M}=0.3$, $\sigma$ is the cross-section of the pair-production process, $\theta=[-\pi,+\pi]$ is the angle between particles, $\eta_{b}$ is the background photon density and $\varepsilon_{t h}$ is the background photon energy threshold as given by Equation 2.3. Due to the nature of the background light in the universe, there are dominant $\eta_{b}$ in different energy regions that need to be addressed to properly estimate $\tau_{\gamma}$. The ones we consider are the extragalactic background light (EBL), for $E_{\gamma}<10^{14.5} \mathrm{eV}$, the cosmic background microwave radiation (CMB) for $10^{14.5} \mathrm{eV}<E_{\gamma}<10^{19} \mathrm{eV}$ and the radio background (RB) for $E_{\gamma}>10^{19} \mathrm{eV}$.

The resulting mean-free path, $\lambda=(c z) /\left(\mathrm{H}_{0} \tau_{\gamma}\right)$, using Eq. 3.1, at the $\mathrm{EBL}^{2}$ and $\mathrm{CMB}$ regions, are presented in the left and right top panels of Fig. 2. For simplicity, we consider that the LIV in the process is dominated by the subluminal photon sector with leading order $n=1$, that is $\delta_{1}^{\text {tot }}=\delta_{\gamma, 1}:=$ $\delta_{1}$, where $\delta_{1}<0$. Once again, the red dotted line in the Figure is the LI expected behaviour and the shaded regions are the none transparent universe as a function of the gamma-ray energy, $E_{\gamma}$, in the different scenarios. The LIV scenarios for (a) $\delta_{1}=\left(-10^{-27},-10^{-28},-10^{-29},-10^{-30}, 0\right) \mathrm{eV}^{-1}$ and (b) $\delta_{1}=\left(-10^{-37},-10^{-38},-10^{-39},-10^{-40}, 0\right) \mathrm{eV}^{-1}$ are shown in the grey areas from darker to lighter, where each area includes the previous one. As can be seen, when LIV is considered, the opacity of the universe change in such a way that allows more photons to arrive from further distances and sources, which have the potential to be measured, which we explore in the next Section. In the bottom of the Figure, there are the energy photon space for each scenario that shows the decrease of the allowed configurations in each different scenario in the top panels. Similar results are expected for $n=0$ and 2 [35].

\subsection{TeV gamma-rays absorption including LIV}

In the region of interest with the EBL dominant background, $\mathrm{TeV}$ gamma-rays are the ones that start to suffer energy loss due pair-production, so, LIV signatures can be expected in the shape of the attenuated spectra of $\mathrm{TeV}$ sources. The left panel of Figure 3 shows the behaviour of the gamma-ray attenuation,

$$
a\left(E_{\gamma}, z ; \delta_{n}^{t o t}, n\right)=\exp -\tau
$$

at $\mathrm{z}=0.03$ and 0.18 , for the scenarios where $\delta_{1}=\left(0,-10^{-27},-10^{-28},-10^{-29},-10^{-30}\right) \mathrm{eV}^{-1}$ and $\tau$ is given by Eq. 3.1. In the red dotted line there is the LI scenario, $\delta_{1}=0$, which has a hard drop in the

\footnotetext{
${ }^{2}$ In the calculations presented here, the Franceschini et al. EBL model is used [43].
} 

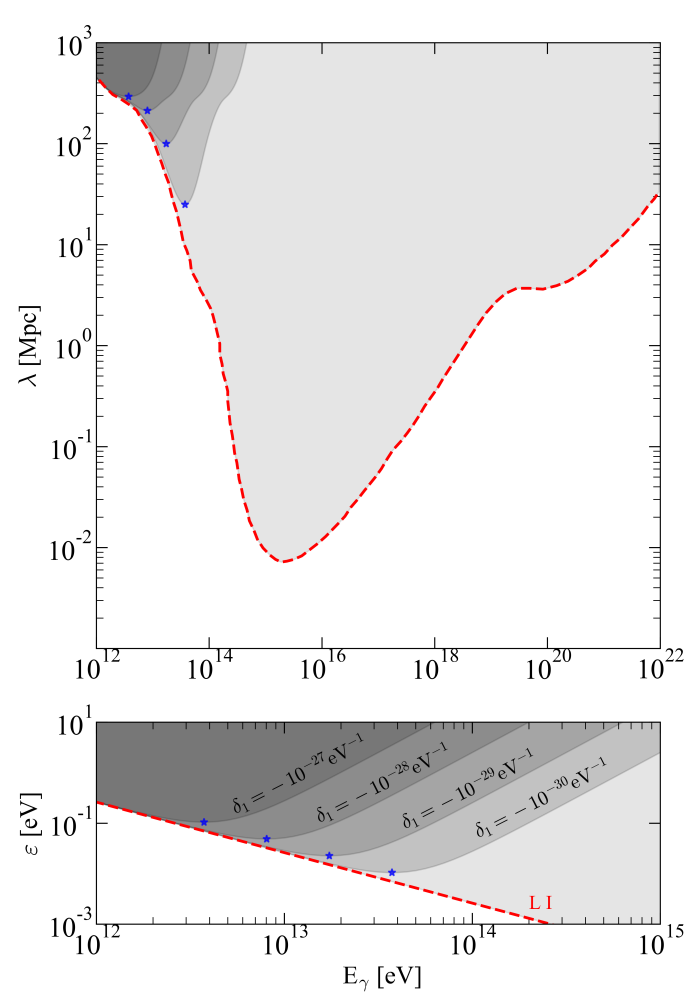

(a)
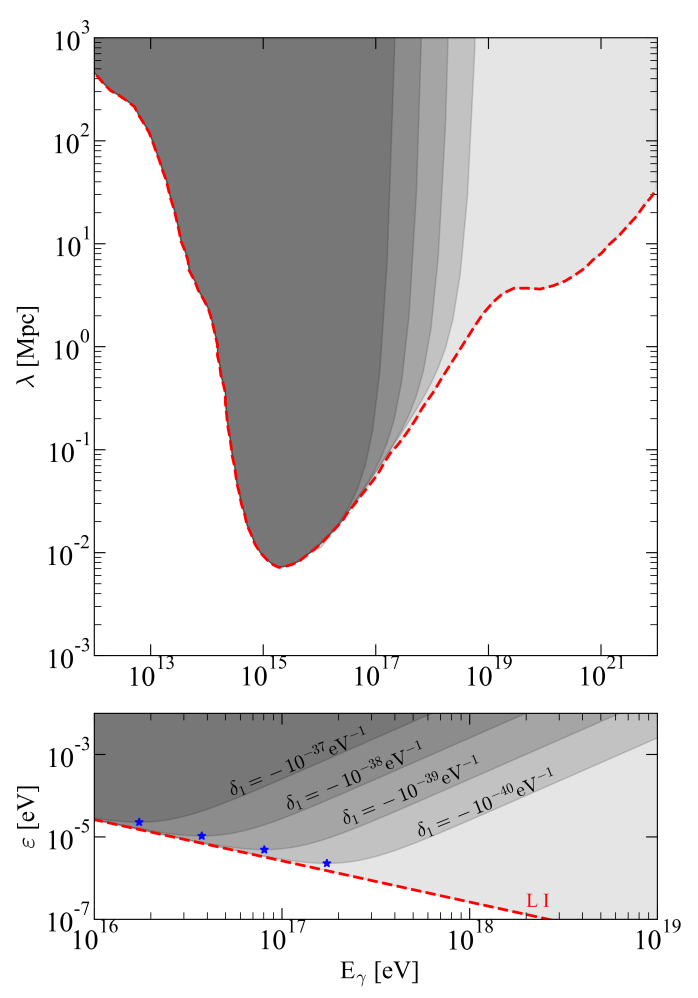

(b)

Figure 2: In the top panels, the photon mean-free path as function of $E_{\gamma}$ at EBL (a) and CMB (b) regions. The red dotted line is the LI expected behaviour $\left(\delta_{1}=0\right)$. The shaded regions are the none transparent universe as a function of the gamma-ray energy, $E_{\gamma}$, in the different scenarios. From the darker to lighter grey: (a) $\delta_{1}=\left(-10^{-27},-10^{-28},-10^{-29},-10^{-30}, 0\right) \mathrm{eV}^{-1}$ and (b) $\delta_{1}=\left(-10^{-37},-10^{-38},-10^{-39},-10^{-40}, 0\right) \mathrm{eV}^{-1}$. Each shaded area includes the previous one. In the bottom panels, allowed regions for the pair production process $\gamma \gamma_{b} \rightarrow e^{+} e^{-}$in the same scenarios that the top panels. Blue stars show the critical values in Eq. 2.5.

attenuation curve. However, when LIV is considered, a recovery in the photon flux can be expected, with an inflection point given by Eq. 2.5, i.e. $\varepsilon^{c r}\left(n, \delta_{n}^{t o t}\right)=\left[\frac{1}{4} \frac{(n+2)^{(n+2)}}{(n+1)^{(n+1)}}\left(\frac{m_{e}^{2}}{4 K(1-K)}\right)^{(n+1)} \delta_{n}^{t o t}\right]^{1 /(n+2)}$ and $E_{\gamma}^{c r}\left(n, \delta_{n}^{t o t}\right)=\left[\frac{4}{n+1} \frac{m_{e}^{2}}{4 K(1-K)} \frac{1}{\delta_{n}^{t o t}}\right]^{1 /(n+2)}$. Shaded areas show where photon events are constrained by the EBL absorption. Each area is inclusive from darker to lighter grey.

In Reference [41], we proposed a new analysis procedure to search LIV signatures in this channel with the most updated $\mathrm{TeV}$ gamma-ray dataset. In there, we look at 111 measured energy spectra from 38 sources, and found that only 18 measured spectra from 6 sources are expected to have significant contribution to restrict the LIV energy scale beyond the current limits to the $E_{L I V}^{(n)}$. We conclude that the dataset is best described by the LI assumption and we set stringent limits to the LIV energy scales at 2, 3 and $5 \sigma(\mathrm{CL}): \mathrm{E}_{\mathrm{LIV}}^{(1)}=\{12.08,9.14,5.73\} \times 10^{28} \mathrm{eV}$ and $\mathrm{E}_{\mathrm{LIV}}^{(2)}=\{2.38,1.69,1.42\} \times 10^{21} \mathrm{eV}$. In addition, it was shown in Ref. [41], that the results are robust under poor knowledge of the EBL, large uncertainties in the intrinsic energy spectra functional form, energy resolution, selection of spectra, and energy bin selection used in the calculation 

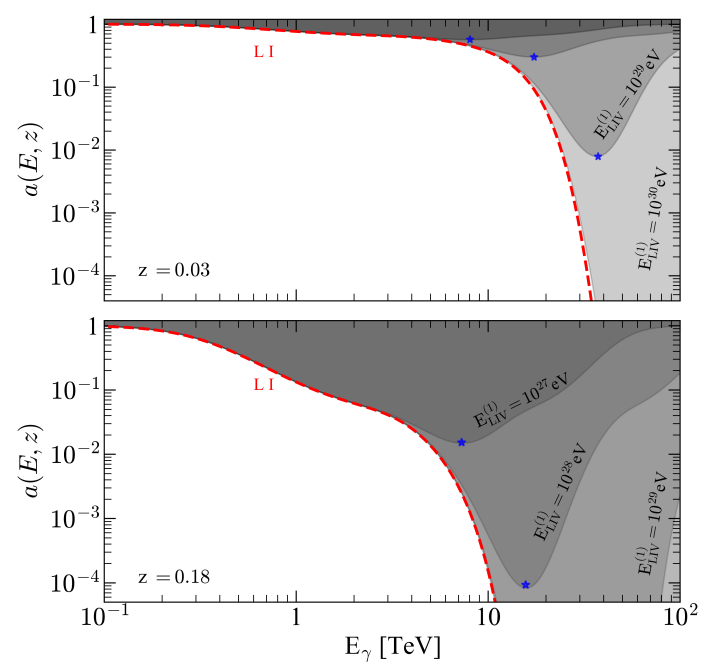

(a)
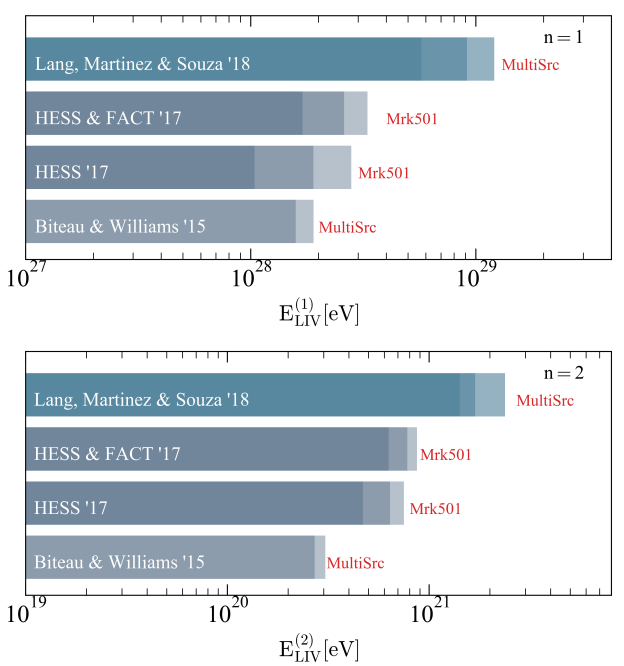

(b)

Figure 3: (a) Gamma-ray attenuation for LI and LIV cases, with $E_{\mathrm{LIV}}^{(1)}=\left(10^{27}, 10^{28}, 10^{29}, 10^{30}\right) \mathrm{eV}$. Blue stars show the critical values given by Eq. 2.5. (b) Comparison of limits imposed on the LIV energy scale. Top panel for $n=1$ and bottom panel for $n=2$. Shades of blues correspond to 2, 3 an $5 \sigma$ CL.

of the intrinsic energy spectra. The right panel in Fig. 3 compares these results with previous limits established by similar LIV signature searches reported in the References [31,36,37].

\subsection{GZK photon flux including LIV}

Kenneth Greisen, Vadim Kuzmin and Georgiy Zatsepin (GZK) showed that UHECR that propagates from further sources have a probability to interact with the CMB and generate a secondary flux of pions via the $\Delta$ resonance, which constrains the propagation distance of UHECR $[44,45]$. Neutral pions decay into a couple of photons most of the times, leading to an expected flux of photons, which are usually named GZK-photons.

As we commented in the previous Sections, the introduction of some LIV can shift the pairproduction energy threshold, which in the subluminal case leads to an increase of the expected photon flux. Thus, in Ref. [35] we proposed a search for LIV signatures at the CMB dominant background region, by computing the GZK-photon flux on Earth considering, for the fist time in the literature, several UHECR injection models and source distribution models. Moreover, Reference [46] shows that the best combination of the two type of models that was shown to best describe the energy spectrum, composition, and arrival direction of UHECR corresponds to the one with a source distribution model that follow a GRB rate evolution proportional to $(1+11 z) /\left[1+(z / 3)^{0.5}\right][47,48]$, and with an injection model that considers a power law energy spectrum at the source with a rigidity cutoff, given by

$$
\frac{d N}{d E_{s}}=\left\{\begin{array}{ll}
E_{s}^{-\Gamma} & , \text { for } R_{s}<R_{\text {cut }} \\
E_{s}^{-\Gamma} e^{1-R_{s} / R_{\text {cut }}}, & \text { for } R_{s} \geq R_{\text {cut }}
\end{array},\right.
$$

where $\Gamma=1.25$ and $\log _{10}\left(R_{\text {cut }} / V\right)=18.5$, and also consider five different species of primary cosmic ray nuclei, with fractions: $f H=0.365, f H e=0.309, f N=0.121, f S i=0.1066$ and 


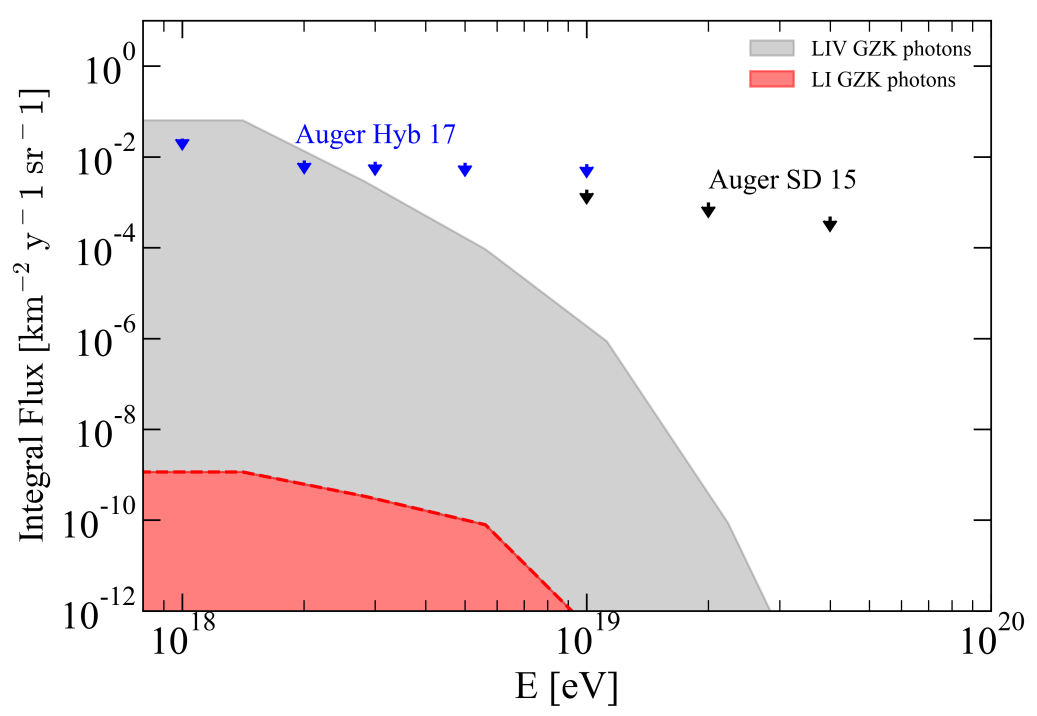

Figure 4: Integral flux of GZK-photons as a function of the photon energy, $E_{\gamma}$, with and without the LIV effects in the model that was shown to best describe the energy spectrum, composition, and arrival direction of UHECR. The arrows correspond to the upper limits to the integrated photon flux from the Pierre Auger Observatory (Auger SD (2015) [50] and Auger Hyb (2017) [51]).

$f F e=0.098$. In Ref. [35] other $\Gamma, R_{\text {cut }} / V$ and fraction values were also used, including a pure proton scenarios and a $\mathrm{Si}$ one, combined with other 4 different source distribution models. The integrated GZK photon flux in the different scenarios were computed with the modified CRPropa3/EleCa codes [49]. As expected, the LIV effect is the increase in the predicted GZK-photon flux, which can be appreciated in Fig. 4. The expected region for the GZK-photons in the LIscenario is the bottom red shaded area and the grey top region shows the one for the GZK-photons including LIV, where the lower limit is the LI-scenario and the top one, where the LIV effect is maximum, $\delta_{n} \rightarrow-\infty$. Comparing this results with the new upper limits of the photon flux obtained by the Pierre Auger Observatory (Auger SD (2015) [50] and Auger Hyb (2017) [51]), in the black and blue arrows, the LIV-scenarios with $\delta_{0} \sim-10^{-20}, \delta_{1} \sim-10^{-38} \mathrm{eV}^{-1}$ and $\delta_{2} \sim-10^{-56} \mathrm{eV}^{-2}$ are compromised. Although, these limits are several orders of magnitude more restrictive than those presented in the previous Section, the comparison is not straightforward due to the different systematics of the measurements and the photon energy region.

\section{Conclusions}

In this proceeding we have reviewed and explored the effects of the phenomenological generalization of the LIV-induced modifications to the dispersion relation via Eq.2.1, that leads, in the subluminal scenario, to an increase in the number of photons that can be expected in the $\mathrm{TeV}$ and EeV energy regions, when compared with the LI case, due to a shift in energy threshold of the pair-production process.

We have presented and discussed the optical depth including significant LIV effects in both regions of interest. In the TeV gamma-ray sector, we have explored the LIV signatures in the TeV spectra and presented stringent limits to the LIV energy scale in the subluminal and photon sector, 
at leading order $n=1$ and 2. At the EeV energy region, we have presented the predicted GZKphoton flux including LIV effects for the scenario that is most compatible with the most updated data of UHECR.

Astroparticle physics has reached the status of precision science due to the development and construction of new observatories, operating innovative technologies and the detection of large numbers of events and sources, which set an unprecedented opportunity to test fundamental physics, such as effects of some Lorentz invariance violation as we have addressed in this proceeding. Moreover, updates and new studies can be expected with the advent of new and better data from the cosmic messengers.

\section{References}

[1] Nambu Y 1968 Supplement of the Progress of Theoretical Physics $\mathbf{E 6 8}$ 190-195

[2] Kostelecky V A and Samuel S 1989 Phys. Rev. D39 683

[3] Colladay D and Kostelecky V A 1998 Phys. Rev. D58 116002 (Preprint hep-ph/9809521)

[4] Ellis J, Mavromatos N E and Nanopoulos D V 1999 Phys. Rev. D 61027503

[5] Gambini R and Pullin J 1999 Phys. Rev. D 59124021

[6] Amelino-Camelia G 2001 Nature 410 1065-1067

[7] Alfaro J 2005 Phys. Rev. Lett. 94221302

[8] Potting R 2013 Journal of Physics: Conference Series Volume 447012009

[9] Audren B, Blas D, Lesgourgues J and Sibiryakov S 2013 JCAP 1308039 (Preprint 1305 . 0009 )

[10] Bluhm R 2014 Springer Handbook of Spacetime chap Observational Constraints on Local Lorentz Invariance, pp 485-507

[11] Calcagni G 2017 Eur. Phys. J. C77 291 (Preprint 1603.03046)

[12] Bettoni D, Nusser A, Blas D and Sibiryakov S 2017 JCAP 1705024 (Preprint 1702 . 07726)

[13] Liberati S and Maccione L 2009 Annual Review of Nuclear and Particle Science 59 245-267 ISSN 0163-8998, 1545-4134 URL

http://www. annualreviews.org/doi/abs/10.1146/annurev.nucl.010909.083640

[14] Coleman S R and Glashow S L 1997 Phys. Lett. B405 249-252 (Preprint hep-ph / 9703240 )

[15] Amelino-Camelia G, Ellis J R, Mavromatos N E, Nanopoulos D V and Sarkar S 1998 Nature 393763 (Preprint astro-ph/9712103)

[16] Coleman S R and Glashow S L 1999 Phys. Rev. D59 116008 (Preprint hep-ph / 9812418)

[17] Stecker F W and Glashow S L 2001 Astropart. Phys. 16 97-99 (Preprint astro-ph/0102226)

[18] Stecker F W 2003 Astropart. Phys. 20 85-90 (Preprint astro-ph/ 0308214 )

[19] Jacobson T, Liberati S and Mattingly D 2003 Phys. Rev. D67 124011 (Preprint hep-ph/0209264)

[20] Stecker F W and Scully S T 2005 Astropart. Phys. 23 203-209 (Preprint ast ro-ph / 012495 )

[21] Ellis J R, Mavromatos N E, Nanopoulos D V, Sakharov A S and Sarkisyan E K G 2006 Astropart. Phys. 25 402-411 [Erratum: Astropart. Phys.29,158(2008)] (Preprint 0712.2781) 
[22] Galaverni M and Sigl G 2008 Phys. Rev. Lett. 100021102 (Preprint 0708 . 1737)

[23] Galaverni M and Sigl G 2008 Phys. Rev. D78 063003 (Preprint 0807 . 1210)

[24] Albert J et al. (MAGIC, Other Contributors) 2008 Phys. Lett. B668 253-257 (Preprint 0708 . 2889)

[25] Stecker F W and Scully S T 2009 New J. Phys. 11085003 (Preprint 0906.1735 )

[26] Xu H and Ma B Q 2016 Astropart. Phys. 82 72-76 (Preprint 1607. 03203)

[27] Chang Z, Li X, Lin H N, Sang Y, Wang P and Wang S 2016 Chin. Phys. C40 045102 (Preprint $1506.08495)$

[28] Ellis J and Mavromatos N E 2013 Astropart. Phys. 43 50-55 (Preprint 1111.1178)

[29] Fairbairn M, Nilsson A, Ellis J, Hinton J and White R 2014 JCAP 1406005 (Preprint 1401.8178 )

[30] Tavecchio F and Bonnoli G 2016 Astron. Astrophys. 585 A25 (Preprint 1510.00980 )

[31] Biteau J and Williams D A 2015 Astrophys. J. 81260 (Preprint 1502.04166)

[32] Martínez-Huerta H and Pérez-Lorenzana A 2017 Phys. Rev. D95 063001 (Preprint 1610.00047 )

[33] Rubtsov G, Satunin P and Sibiryakov S 2017 JCAP 1705049 (Preprint 1611.10125 )

[34] Martínez-Huerta H (HAWC) 2018 PoS ICRC2017 868 (Preprint 1708. 03384 )

[35] Guedes Lang R, Martínez-Huerta H and de Souza V 2018 Astrophys. J. 85323 (Preprint 1701.04865 )

[36] Cologna G et al. (FACT, H.E.S.S.) 2017 AIP Conf. Proc. 1792050019 (Preprint 1611.03983 )

[37] Lorentz M and Brun P (H.E.S.S.) 2017 EPJ Web Conf. 13603018 (Preprint 1606.08600 )

[38] Pfeifer C 2018 Phys. Lett. B780 246-250 (Preprint 1802.00058 )

[39] Ellis J, Konoplich R, Mavromatos N E, Nguyen L, Sakharov A S and Sarkisyan-Grinbaum E K 2018 (Preprint 1807.00189)

[40] Abdalla H and BÃúttcher M 2018 Astrophys. J. 865159 (Preprint 1809.00477 )

[41] Lang R G, Martínez-Huerta H and de Souza V 2018 (Preprint 1810.13215)

[42] De Angelis A, Galanti G and Roncadelli M 2013 Mon. Not. Roy. Astron. Soc. 432 3245-3249 (Preprint 1302.6460)

[43] Franceschini A, Rodighiero G and Vaccari M 2008 Astron. Astrophys. 487837 (Preprint 0805.1841 )

[44] Greisen K 1966 Phys. Rev. Lett. 16(17) 748-750

[45] Zatsepin G T and Kuz'min V A 1966 Soviet Journal of Experimental and Theoretical Physics Letters 478

[46] Unger M, Farrar G R and Anchordoqui L A 2015 Phys. Rev. D92 123001 (Preprint 1505.02153 )

[47] Le T and Dermer C D 2007 The Astrophysical Journal 661394

[48] Kotera K, Allard D and Olinto A V 2010 JCAP 1010013 (Preprint 1009.1382)

[49] Batista R A, Dundovic A, Erdmann M, Kampert K H, Kuempel D, MÃijller G, Sigl G, van Vliet A, Walz D and Winchen T 2016 Journal of Cosmology and Astroparticle Physics 2016038

[50] Carla Bleve for the Pierre Auger Collaboration 2015 Procedings of Science (ICRC2015) 1103 URL https://pos.sissa.it/236/1103/pdf

[51] The Pierre Auger Collaboration 2017 Journal of Cosmology and Astroparticle Physics 2017009 URL http://stacks.iop.org/1475-7516/2017/i=04/a=009 